\title{
Impact of Driver Compliance on the Safety and Operational Impacts of Freeway Variable Speed Limit Systems
}

Bruce Hellinga ${ }^{1}$, PhD, PEng and Michael Mandelzys ${ }^{2}$

1. Associate Professor

(Corresponding Author)

Department of Civil and Environmental Engineering, University of Waterloo

Waterloo, Ontario, N2L 3G1, Canada

Tel: (519) 888-4567 ext. 2630

Fax: (519) 888-4349

E-mail: bhellinga@uwaterloo.ca

\author{
2. MASc Candidate \\ Department of Civil and Environmental Engineering, \\ University of Waterloo \\ Waterloo, Ontario, N2L 3G1, Canada \\ Tel: (519) 888-4567 ext. 33872 \\ Fax: (519) 888-4349 \\ E-mail: mamandel@uwaterloo.ca
}

Accepted for publication in the ASCE Journal of Transportation (Feb. 2010) 


\section{ABSTRACT}

Variable Speed Limit (VSL) systems enable freeway system managers to dynamically change the posted speed limit on a section of roadway in response to varying conditions. VSL system goals may include homogenizing traffic flow, improving safety, and/or reducing driver stress. Although it is understood that the effectiveness of VSL systems is impacted by the level of driver compliance, which itself is influenced by the extent of speed limit enforcement, very little is known about the strength of these impacts. This paper makes use of a simulation model to evaluate the sensitivity of the safety and operational impacts of VSL to driver compliance.

Several scenarios for driver compliance were modeled using the PARAMICS microscopic traffic simulator. Findings indicated that VSL impacts are very sensitive to the level of driver compliance. Safety was shown to be positively correlated with the level of compliance and travel time was shown to be negatively correlated. However, it was also found the magnitude of the impact is strongly influenced by the VSL control strategy (i.e. set of rules for incrementing and decrementing the speed limits) being used. Therefore, selection of the VSL control strategy cannot be done independently of the decision regarding speed limit enforcement.

\section{INTRODUCTION}

Variable Speed Limit (VSL) systems consist of changeable message signs (CMSs) deployed along the freeway and connected via a communication system to a traffic management centre. The CMSs, rather than traditional static signs, are used to display the regulatory or advisory speed limit, enabling freeway system managers to dynamically change the posted speed limit in response to prevailing traffic and/or weather conditions. In general, VSL systems attempt to homogenize traffic flow, improve safety, and reduce driver stress. 
Special purpose VSL systems, such as those that respond to inclement weather have been in use in North America and elsewhere for many years (e.g. fog response system on I-75 in Tennessee; winter weather response system on I-90 through the Snoqualmie Pass in Washington; winter weather response system on E-18 motorway in Finland). These systems typically operate by sensing adverse weather conditions and then responding by decreasing the posted speed limit to pre-specified levels. Often the displayed speed is augmented with a notification of the weather conditions that has prompted the reduced speed.

General purpose VSL systems have also been deployed in many jurisdictions outside of North America, including Britain, the Netherlands, Germany, Australia, Austria, New Zealand and Israel. The deployment of VSL system in North America has been much more limited but appears to be increasing with recent deployments on the I-495 in Delaware, I-270 and I-255 in Missouri (MODOT, 2009), and I-90 and I-5 in Washington (WSDOT, 2009).

Despite the relatively large number of VSL field deployments, there remains limited evidence (empirical or otherwise) that quantifies the impact that VSL has on safety and traffic flow. Several recent publications provide good reviews of the existing literature related to impacts of VSL (Lee and Abdel-Aty, 2008; Abdel-Aty et al., 2008; Allaby et al., 2007). Much of the focus of VSL system evaluation studies has been on safety. The general consensus that emerges from these studies is that VSL can provide safety benefits, particularly in the reduction of rear-end collisions. However, there is also evidence (some of it anecdotal) that the safety benefits provided by VSL are highly dependent on the VSL control strategy employed (e.g. frequency and conditions under which speed limits are increased and decreased), the traffic conditions, and level and type of enforcement (Abdel-Aty et al., 2008). 
There appears to be even less evidence to suggest that VSL increases traffic flow efficiency. Papageorgiou et al (2008) used data from a German VSL controlled motorway to investigate the impact that VSL has on aggregate traffic stream behaviour and therefore traffic flow efficiency. The authors found that their data analysis was inconclusive regarding the potential for VSL to increase flow capacity. However, they also observed that their data analysis was constrained by the conditions that existed at the study site (including the VSL control algorithm and extent of enforcement) and they suggested that a more robust and efficient VSL control algorithm could be developed.

The body of literature on the impacts of VSL seems to suggest:

1. VSL systems have the potential to provide significant safety benefits.

2. There is little evidence to suggest that VSL increases capacity flow and safety benefits may be achieved at the expense of increasing travel times.

3. VSL impacts are influenced by the driver compliance to the posted speed limits.

In most jurisdictions, driver compliance with posted speed limits is highly influenced by the type and extent of speed limit enforcement (Povey et al, 2003; Benekohal et al, 2008; TRB, 1998). Consequently, a number of VSL systems (e.g. Britain, Netherlands) employ automated speed enforcement. However, in North America, unlike many other parts of the world, automated speed enforcement is not widely used. Rodier et al. (2007) report that as of 2007, automated speed enforcement programs operated in the U.S. in only 11 states and in Washington D.C., and that most of these were located on residential streets and not highways. In September 2008 Arizona deployed a state-wide highway speed limit enforcement program that is currently the largest program in North America. The lack of greater use of automated speed enforcement on highways in North America can be attributed to several factors including legal restrictions 
(including constitutional, legislation, and evidentiary issues) and lack of strong support from the general public and elected officials.

In North America, there is increasing interest to deploy VSL system on freeways and at the same time there appears to be barriers to the use of automated speed enforcement. Within this context, the influence of driver compliance on the effectiveness of VSL is particularly important. Unfortunately, very little is known about the influence of driver compliance on the impacts of VLS systems.

In this paper, we focus on the influence that driver compliance has on the safety and operational impacts of VSL. Specifically, we make use of a simulation model to evaluate the sensitivity of the safety and operational impacts of VSL to driver compliance. The next section discusses driver compliance to speed limits, and in particular identifies relevant studies from the literature and then describes an empirical investigation on driver compliance conducted as part of this study. The middle sections describe the approach used to model driver compliance to variable speed limits and the VSL evaluation approach and results. The final section presents the conclusions of the study.

\section{DRIVER REACTION TO SPEED LIMITS}

\section{Evidence from the literature}

There is relatively little work in the literature describing driver compliance to variable speed limits.

Ulfarsson et al (2005) evaluated the impact of variable speed limits posted on I-90 in the vicinity of Snoqualmie Pass, Washington State using empirical data. The study concluded that the use of variable speed limits caused a statistically significant reduction in mean vehicle speeds but no 
consistent impact on variation on speed was found. Furthermore, driver response to this VSL system is not likely transferable to general VSL systems given the unique nature of the Snoqualmie Pass geometry, terrain, and weather.

Park and Yadlapati (2003) conducted a simulation study of VSL in work zones in Virginia using VISSIM. In their study they considered three different levels of speed compliance in which $70 \%$, $80 \%$ or $100 \%$ of the vehicles complied with the posted speed. No evidence was given to indicate which of these three compliance levels is most likely to be achieved in practice nor was the distribution of speeds associated with a given posted speed limit described.

A more recent study (Riffkin et al., 2008) examined speed compliance in response to VSL in a work zone on the I-80 in Utah. The study found that both mean speed and variation of speeds were reduced as a result of VSL. However, in this study, speed limits were varied only twice per day (65 mph at night and $55 \mathrm{mph}$ from 7 am to $6 \mathrm{pm}$ ). Distributions of speed were generated, however, these distributions included congested conditions as well as uncongested conditions and therefore the distributions do not solely reflect drivers' response to the posted speed limit.

Lee and Abdel-Aty (2008) used a driver simulator to examine the behaviour of 86 participants as they drove an $8 \mathrm{~km}$ freeway section along which they encountered variable message signs (VMS) warning of downstream speed changes implemented via VSL. The study showed that the presence and type of message displayed on the VMS had a statistically significant impact on the level to which drivers complied with the downstream speed.

In 1992, a 20-km section of the A2 motorway between Amsterdam and Utrecht in the Netherlands was outfitted with a variable speed limit system. The system allowed speeds to be set for each lane individually, and to be lowered incrementally from $120 \mathrm{~km} / \mathrm{h}$ to $90 \mathrm{~km} / \mathrm{h}$ or to $70 \mathrm{~km} / \mathrm{h}$. A before-and-after evaluation study was conducted (van den Hoogen and Smulders, 
1994) to assess impacts including driver behaviour. The study found that on average speeds declined after implementation of VSL. However, the analysis did not consider only free flow vehicle speeds and therefore the compliance to VSL cannot be inferred. Speed variation was also reported to decrease by an unspecified amount and the study reported that "the speed signs were complied with by the drivers", but this finding is not numerically quantified within the paper. Note that the study did not outline what enforcement measures were used in conjunction with the variable speed limit signs.

Another before-and-after study was carried out on the M25 "Controlled Motorway" in Britain (UK Highways Agency, 2006).The study found that there was an increase in compliance with the speed limit following the introduction of VSL, but this increase is not quantified. It should be noted that speed limit compliance on the M25 is automatically enforced via cameras mounted to overhead gantries.

In 2006, a variable speed limit system was implemented at a work zone for a three week period in Twin Cities, Minnesota. Kwon et al. (2007) outline the development of the VSL control strategy and the results of the implementation. The results of the implementation were evaluated as a before-and-after study using data on speed variation, total throughput, and traffic response.

To assess driver compliance, Kwon et al. correlated the difference between vehicle speed and the posted speed on the VSL they were about to encounter versus the change in speed from before the VSL was seen to after the VSL was seen. If all drivers complied with the VSL, the two numbers should be approximately equal. The correlation coefficient found over different days ranged between $20 \%$ and $60 \%$. It was also observed that the correlation was weaker as the difference between the actual traffic speed and posted speed limit increased suggesting that 
drivers are less likely to comply with the variable speed limit if the posted speed is significantly different from the speed they would otherwise choose. It is important to note that in addition to being a work zone, the variable speed limit system evaluated in this paper used advisory limits only.

A study conducted in 1999 and 2000 (Giles, 2004) collected more than 3.3 million spot-speed measurements of vehicles on 194 roadways in Western Australia. Driver compliance to the static posted speed limit was determined by considering speeds only from free flow vehicles ${ }^{1}$. The resulting data, segregated by posted speed limit, were found to follow a near-Normal distribution. The mean free flow speed, standard deviation of free flow speed, and percent free flow vehicles exceeding the posted speed limit are provided in Table 1.

The study found that though a significant portion of free flow vehicles exceeded the posted speed limit for all posted speed limits, the average free flow speed was typically less than or equal to the posted speed limit and the coefficient of variation (standard deviation divided by the mean) ranged from 0.18 to 0.13 with an average of approximately 0.15 .

In summary, it appears that there is no empirical data that quantitatively describes driver compliance to variable speed limits for general purpose VSL systems. There is data describing driver compliance with static speed limits (i.e. Giles, 2004 study) but it is not clear that these data characterize North American drivers and there is evidence to suggest that drivers respond differently to static speed limits than to VSL (i.e. Kwon et al., 2007).

In response to these findings, an analysis was conducted to determine driver compliance to static speed limits on an urban freeway in North America.

\footnotetext{
${ }^{1}$ Free flow vehicles were defined in this study as those with a time headway $\geq 4$ seconds.
} 
Driver compliance to static speed limits on an urban freeway in Ontario, Canada

The study described by Giles (2004) made use of spot-speed data collected from dedicated speed studies. Such studies are resource intensive, especially when large sample sizes are desired. Furthermore, our interest is restricted to urban freeways, which are already typically instrumented with loop detectors (or comparable sensors). Consequently, we elected to develop measures of speed compliance from vehicle speeds measured by dual loop detectors.

The study site consisted of the eastbound direction of a $10 \mathrm{~km}$ section of an urban freeway, the Queen Elizabeth Way (QEW), located near Toronto, Canada (Figure 1). The section has three mainline lanes, four interchanges, a static posted speed limit of $100 \mathrm{~km} / \mathrm{h}$ and experiences a directional AADT of about 70000 vehicles. The section is instrumented with loop detector stations each consisting of dual loop detector groups in each travel lane. These loops measure speed, volume, and occupancy, and report aggregate results every 20-seconds.

Loop detector reported speeds are an average of the speeds of individual vehicles passing the loop during the 20 second polling interval. Speed compliance cannot be determined from aggregated data nor can free flow data be discerned from aggregated data. Consequently, speed data were only used from intervals in which only a single vehicle was observed.

Data were obtained from the Ministry of Transportation of Ontario for each loop detector over two days from 12:00 midnight until 9:00 a.m. The overnight time period was selected to ensure that (a) the highway was free-flowing, and (b) individual vehicle speeds could be obtained from the loop detector data.

The data were also filtered to remove very high (greater than $170 \mathrm{~km} / \mathrm{h}$ ) and very low (less than $60 \mathrm{~km} / \mathrm{h}$ ) speeds. This was done to reduce the influence of measurement error, accelerating 
vehicles, and extreme outliers on the speed profile. The resulting free-flow speed profile is provided in Figure 2.

The speed profile from Figure 2 has a mean of $119 \mathrm{~km} / \mathrm{h}$, a standard deviation of $13.1 \mathrm{~km} / \mathrm{h}$, and a coefficient of variation of 0.11 . The distribution appears to be generally Normally distributed (as indicated by the solid line in Figure 2); however statistical testing suggests that the distribution of the loop detector data is slightly skewed to the right (i.e. higher than expected proportions of vehicles travelling at high speeds). Nevertheless, these differences are relatively small and it is assumed that free speeds can be best represented by a Normal distribution.

These results can be compared to those obtained by the Western Australian speed study for roadways with a posted speed limit of $100 \mathrm{~km} / \mathrm{h}$ (Giles, 2004).

- The mean free flow speeds are significantly different (Australia: $93.8 \mathrm{~km} / \mathrm{h}$; Toronto: 119 $\mathrm{km} / \mathrm{h})$.

- Despite the rather large difference in the mean free flow speeds, the variability of free flow speeds relative to the mean are similar (COV Austrian data $=0.13$; COV Toronto data $=0.11)$.

- The Australia data indicates a much higher level of speed compliance ( $70 \%$ observations $\leq$ the posted speed limit) than does the Toronto data (only 5\%).

These differences may result from differences in enforcement, roadway characteristics, legislation (process and penalties) associated with speeding, or with definition of free flow. The Australian study used a time headway threshold of 4 seconds to define free flow vehicles. This study considered only speed measurements associated with a single vehicle in a 20 second interval. If the traffic stream can be assumed to follow a Poisson process (headways are exponentially distributed), then from Figure 3 we can see that using the criteria that headways $\geq$ 
4 seconds constitutes free flow will result in a much larger proportion of the vehicles in the traffic stream being included than using the criteria of one observation per 20 second polling interval. This also implies that the free speed data from the Australian study included observations from much higher traffic volumes than the Toronto data. Consequently, even if these vehicles had time headways greater than 4 seconds, their choice of speed may have been impacted (i.e. reduced) by the presence of other vehicles on the roadway, and therefore, overrepresent level of compliance.

\section{MODELLING DRIVER COMPLIANCE TO SPEED LIMITS}

The previous section quantified observed driver response to a fixed (static) speed limit of 100 $\mathrm{km} / \mathrm{h}$. However, we still have insufficient evidence to reliably predict how drivers will response to VSL. Consequently, we define four possible driver reactions (levels of compliance) to variable speed limits and then examine the sensitivity of the VSL performance to these levels.

We consider four levels of compliance: Low, Moderate, High, and Very High (Table 2). For all four levels of compliance, free flow speeds are assumed to remain Normally distributed as was observed from driver compliance to static speed limits.

These compliance relationships are illustrated graphically in Figure 4.

\section{Low Compliance:}

The Low compliance scenario represents a condition in which drivers react to a VSL of $100 \mathrm{~km} / \mathrm{h}$ as they do currently with a static speed limit of $100 \mathrm{~km} / \mathrm{h}$ (i.e. with a mean free speed that is $19 \%$ higher than the posted speed limit and only approximately $5 \%$ of free speeds are the posted speed limit). However, for VSL speed limits of less than $100 \mathrm{~km} / \mathrm{h}$ (i.e. 80 and $60 \mathrm{~km} / \mathrm{h}$ ), 
we assume the compliance rate decreases resulting in mean free speeds of 104 and $88 \mathrm{~km} / \mathrm{h}$ respectively (corresponding to compliance rates of approximately $1 \%$ and $0.1 \%$ respectively).

\section{Moderate Compliance:}

The Moderate compliance scenario coincides most closely with the level of speed compliance observed on the QEW. Observed data indicate that for a posted static speed limit of $100 \mathrm{~km} / \mathrm{h}$, the mean free speed is $119 \mathrm{~km} / \mathrm{h}$ or $19 \%$ higher than the posted speed limit and approximately $5 \%$ of vehicles adopt a free speed less than or equal to the speed limit. We have assumed that under this level of compliance, the mean free flow speed is expected to remain $19 \%$ higher than the posted speed limit (i.e. $95 \mathrm{~km} / \mathrm{h}$ and $71 \mathrm{~km} / \mathrm{h}$ for posted limits of $80 \mathrm{~km} / \mathrm{h}$ and $60 \mathrm{~km} / \mathrm{h}$ respectively) and approximately $6 \%$ of vehicles adopt a free speed less than or equal to the posted speed limit.

\section{High Compliancee:}

The High compliance scenario assumes that drivers will adopt free speeds that are more closely aligned with the VSL than they do in response to static speed limits. We assume that approximately $25 \%$ of drivers adopt a free speed that is less than or equal to the speed limit. Instead of a mean free speed of $19 \%$ higher than the posted speed limit (as is exhibited for existing field conditions), it is assumed that the mean free speed will be only $8 \%$ higher than the posted speed limit.

\section{Very High Compliance:}

The Very High compliance scenario assumes that approximately $70 \%$ of drivers adopt free flow speeds that are less than or equal to the posted speed limit (the level of compliance observed in 
the Australian speed study (Giles, 2004) for static speed limits of $100 \mathrm{~km} / \mathrm{h}$ ). The resulting average free flow speed is equal to approximately $95 \%$ of the posted speed limit.

\section{VSL SENSITIVITY TO SPEED LIMIT COMPLIANCE}

\section{Simulation Framework:}

The microscopic traffic simulator PARAMICS was selected to perform the modeling work. PARAMICS was chosen primarily because it allows the user to implement custom control logic via an Application Programming Interface (API). Through the API, the user-defined VSL control algorithm overrides the standard code in PARAMICS to dynamically change link- based speed limits.

The study site was the $10 \mathrm{~km}$ section of the QEW depicted in Figure 5. The QEW services a large volume of commuter traffic in the morning and evening peak periods, resulting in heavy congestion and a high frequency of crashes.

The modelled segment was coded using actual geometry and traffic volume data. An origindestination (O-D) matrix was estimated from morning peak-period (6 am to $10 \mathrm{am}$ ) loop detector data averaged over 15 non-incident weekdays. Also, temporal variations in volume were examined to estimate the temporal release profile for each O-D pair. Dual loop detectors were placed in the modelled network at approximately the same locations as those in the field and were programmed to report 20-second speed, volume and occupancy data. A "base model" was established upon validation of existing (non-VSL) conditions, based on temporal speed profiles produced from both observed and simulated data for each detector station. Simulation parameters were adjusted until the speed profiles adequately (within confidence limits of + /$2 \sigma$ ) matched the observed profiles. This Paramics model was previously utilized in a paper by 
Allaby et. al. (2007), and a detailed description of the model calibration/validation procedure can be found within that paper.

The VSL system infrastructure was represented within PARAMICS by thirteen VSL signs placed throughout the network. Each VSL sign was placed next to a loop detector and spaced at approximately $500 \mathrm{~m}$ to $600 \mathrm{~m}$. Since PARAMICS assigns speed limits by link, the mainline was coded as a series of links corresponding to each detector-VSL sign pair.

The VSL control strategy tested in the simulation was designed to select an appropriate speed limit on the basis of 20-second speed, volume, and occupancy loop detector data. This preserved the potential for practical application of the control strategy. Based on predetermined parameter (threshold) values, each combination of speed, volume, and occupancy data represented a particular speed limit decision. The control strategy to select a speed to display is presented in Figure 6.

Once a speed is determined at the current location, speeds may be altered in upstream segments in order to provide a smooth transition between speed regimes (i.e. avoid transitioning directly from $100 \mathrm{~km} / \mathrm{h}$ to $60 \mathrm{~km} / \mathrm{h}$ ). For brevity, the specific transition zone logic is not described in this paper but is described in detail in the literature (Allaby, 2006; Allaby et al., 2007).

Once a reduction in posted speed occurs, the speed cannot be incremented until traffic flow has improved. The required improvement was specified as three consecutive cycles (i.e. 1 minute) of detected station occupancy less than or equal to $15 \%$. The value of $15 \%$ was chosen since traffic plots indicate it is approximately the threshold of flow breakdown.

The crash model structure employed in this study was first introduced by Lee et al. in 2003 . The model uses a calibrated log-linear function to determine a relative crash potential based on 
exposure, control factors, and categorized levels of time varying traffic conditions. These traffic conditions, termed crash precursors, are related to the turbulence experienced within a traffic stream. More turbulent levels of crash precursors correspond to a higher likelihood of an impending crash situation. The model was calibrated through log-linear regression to find a disparity between precursors that exist prior to a crash and those that exist during non-crash conditions. The general form of the crash prediction model developed by Lee et. al (2003) is presented in the following equation. Traffic data for crash conditions were compiled from loop detector data preceding 299 crashes on the QEW between 1998 and 2003.

$\ln (F)=\theta+\Sigma\left(\lambda_{\text {crash_precursors }}\right)+\Sigma\left(\lambda_{\text {control_factors }}\right)+\beta \ln (E X P)$

Where $F$ is the crash frequency, $\theta$ is a constant, $\lambda_{\text {crash_precursors }}$ are values related to turbulence in the traffic stream, $\lambda_{\text {control_factors }}$ are factors to control for the effects of road geometry and peak/off-peak conditions, EXP is the exposure in vehicle-kilometres of travel, and $\beta$ is the parameter for the exposure.

Crash Potential (CP) is calculated by dividing the crash frequency by exposure as per the following equation.

$C P=\left(F / E X P^{\beta}\right)$.

Microsimulation based crash models that evaluate safety by looking for conditions that are precursors to increased accident likelihood, have been utilized in much recent literature. Some examples include Allaby et. al. (2007) and Cunto and Saccomanno (2008).

The advantage of this crash model is that it can provide a dynamic relative measure of crash risk with changing traffic conditions by being updated as often as new traffic data becomes available (i.e. 20 second loop detector intervals). Also, the model can capture the spatial or temporal changes in crash risk which may exist between adjacent road sections based on the introduction 
of a traffic control/management system such as VSL. In this study, the safety impact of VSL was measured by calculating the relative change in CP from the non-VSL case to the VSL case for each compliance scenario an indicated in the following equation.

Relative Change in Safety $=\left(C P_{\text {Non-VSL }}-C P_{V S L}\right) / C P_{\text {Non-VSL }}$

Where $C P_{\text {Non-VSL }}$ is the average crash potential on the roadway before VSL is implemented and $C P_{\text {VSL }}$ is the average crash potential on the roadway after VSL is implemented.

Results:

The PARAMICS simulation model was used to simulate the AM Peak period under all four speed limit compliance scenarios. Ten runs, each with a different random number seed, were conducted for each compliance scenario. The impacts of VSL were quantified in terms of safety (increase is desirable) and travel times (increase is undesirable). The results are provided in Figure 7.

Interpretation of Results:

A number of observations can be made on the basis of the results in Figure 7:

- Compliance level has a very significant impact on VSL safety performance. As expected, benefits increase (though non-linearly) as compliance level increases. The largest increase in safety occurs for the change from Low to Moderate compliance. Increases in safety become smaller as compliance increases. Nevertheless, safety continues to improve for all increases in speed limit compliance.

- Compliance level also has an influence on VSL travel time impacts. As expected, travel times increase as compliance level increases. The impact on travel time is relatively modest for the Low, Moderate and High compliance scenarios as the change in travel 
time with changes in level of compliance is small. However, for the Very High compliance scenario, the increase in travel time is very large. This result might suggest that there is little incentive to implement VSL in a way that attempts to achieve very high speed limit compliance, as the additional safety benefit is small and the cost in terms of increased travel time is very large. However, this result is unexpected and somewhat counter-intuitive. It was speculated that these results reflected characteristics of the VSL strategy evaluated (and the VSL parameter values) rather than VSL per se. More specifically, it was hypothesized that under the Very High compliance level, the existing VSL strategy would result in a lowering of the speed limit to $60 \mathrm{~km} / \mathrm{h}$ (the minimum permitted), but would not subsequently increase the speed limit since increase conditions were rarely met (i.e. loop detector occupancy less than $15 \%$ for three consecutive 20-second intervals). This hypothesis was confirmed through a detailed examination of the simulation results.

Figure 8 illustrates results for a single detector station (Station 100 in Figure 5) from a single simulation run for the Very High speed compliance scenario. As expected, average vehicles speeds are always less than the posted speed limit and the posted speed limit decreases from the initial value of $100 \mathrm{~km} / \mathrm{h}$ to $60 \mathrm{~km} / \mathrm{h}$ in accordance with the VSL strategy. However, speed limits rarely increment. The condition that detector occupancy be less than $15 \%$ for three consecutive 20-second intervals is experienced only 11 times during the entire simulation (circular markers on Figure 8). Furthermore, even when this condition is met, speeds may not increment because of other constraints (e.g. speed reduction conditions defined in Figure 6). This suggests that when compliance is very high, the VSL strategy is able to decrease the speed limit, but is not able to increment the speed limit again. Consequently, implemented speed limits are often lower than required, resulting in unnecessary increases in vehicle travel times. 
As a result of these observations, additional simulation runs were conducted to determine the performance of VSL under the Very High compliance scenario when the occupancy threshold for incrementing speed limits was changed from $15 \%$ to $20 \%$. All other attributes of the simulation were held constant.

The results in Figure 9 indicate that when the occupancy threshold is changed from $15 \%$ to $20 \%$, the number of instances in which the occupancy threshold conditions for increasing the speed limit is 140 (as compared with just 11 when the occupancy threshold is $15 \%$ ). However, the overall impact on the posted speed limit is insignificant. It appears that a change in occupancy threshold alone is insufficient to change the VSL operations. Consequently, additional simulations were carried out in which the occupancy threshold was changed from $15 \%$ to $20 \%$ and the speed limit threshold for decreasing speeds was reduced by $10 \mathrm{~km} / \mathrm{h}$ (i.e. $80 \mathrm{~km} / \mathrm{h}$ was changed to $70 \mathrm{~km} / \mathrm{h}$; and $60 \mathrm{~km} / \mathrm{h}$ was changed to $50 \mathrm{~km} / \mathrm{h}$ ). Figure 10 illustrates the results. For these conditions the speed limits increment more frequently and are sustained at higher speed limits for longer periods.

Figure 11 provides the safety and travel time impacts for these three Very High compliance scenarios averaged over 10 simulation runs. These results indicate that safety and travel time impacts are dependent on the parameter values chosen for the VSL strategy. This finding is consistent with the results of the previous VSL study (Allaby et al., 2007). However, this finding also demonstrates that the most appropriate (optimal) set of parameter values is a function of the level of speed limit compliance exhibited by drivers. Furthermore, these results suggest that the safety benefits associated with the Very High compliance scenario as indicated in Figure 7 are modestly under-estimated and the travel time increases are significantly over-estimated. 
Caveats:

All four speed limit compliance scenarios evaluated in this study assume that the coefficient of variation of the free flow speed remains constant. This implies that the variation in driver behaviour with respect to speed limit compliance remains proportional to the mean speed. It can be reasonably expected that as the level of speed limit compliance increases, the ratio of the standard deviation of free flow speed to the mean speed (i.e. COV) would decrease. This is particularly true for the Very High speed limit compliance scenario. For example, if automated enforcement were in effect, it is reasonable to expect all drivers to select a speed very near to the posted speed limit - especially for speed limits of less than $100 \mathrm{~km} / \mathrm{h}$. If the COV of free flow speed is less than $10 \%$ then we expect increased safety benefits and smaller travel time penalties. Consequently, this can be interpreted to mean that the results obtained in this study for the Very High level of speed limit compliance represent a conservative estimate (i.e. estimated results represent a lower bound on the expected safety benefits and an upper bound on the travel time penalty) and for the Low compliance scenario, the results represent an optimistic estimate.

It must also be noted that altering speed limit compliance with static speed limits will also impact safety and travel times. This study has not separated the relative contribution that speed limit compliance and VSL have on safety and travel time impacts.

\section{CONCLUSIONS}

This study has examined driver compliance to posted speed limits and the subsequent impact that level of compliance has on the operational and safety performance of variable speed limit (VSL) systems. The study findings are as follows: 
1. There appears to be very little empirical evidence reported in the literature that quantifies driver compliance to posted speed limits and in particular variable speed limits.

2. There is also very limited evidence reported in the literature that quantifies the operational and safety impacts of VSL systems, particularly those that do not also implement automated speed enforcement.

3. VSL impacts, in terms of safety and travel times, are quite sensitive to the level of speed compliance. The safety benefits of VSL under the Very High compliance scenario modelled in this study are more than 4 times the benefits obtained under the Low compliance scenario.

4. Safety benefits of VSL increase with increasing speed limit compliance.

5. Travel time penalties (i.e. increases) that result from VSL also increase with increasing speed limit compliance.

6. The optimal VSL strategy and set of parameter values are influenced by the level of speed compliance. Consequently, selection of VSL operating strategy (and parameter levels) cannot be done independent of the decision regarding speed limit enforcement.

\section{REFERENCES}

Abdel-Aty, M., Cunningham,J., Gayah, V.V., and Hsia, L. (2008). “Dynamic Variable Speed Limit Strategies for Real-Time Crash Risk Reduction on Freeways". Transportation Research Record: Journal of the Transportation Research Board, No. 2078, Transportation Research Board of the National Academies, Washington, D.C., 2008, pp. 108-116. 
Allaby, P. (2006). "An Evaluation of the Safety and Operational Impacts of a Candidate Variable Speed Limit Control Strategy on an Urban Freeway". Masters Thesis, University of Waterloo, Civil Engineering, Waterloo.

Allaby, P., Hellinga, B., \& Bullock, M. (2007). "Variable Speed Limits: Safety and Operational Impacts of a Candidate Control Strategy for Freeway Applications". IEEE Transactions on Intelligent Transportation Systems , 8 (4), 671-80.

Benekohal, R.F., Chitturi, M.V., Hajbabaie,A., Wang, M.H., and and Medina, J.C. (2008). "Automated Speed Photo Enforcement Effects on Speeds in Work Zones". Transportation Research Record: Journal of the Transportation Research Board, No. 2055, Transportation Research Board of the National Academies, Washington, D.C., 2008, pp. 11-20.

Cunto, F., \& Saccomanno, F. (2008). "Calibration and Validation of Simulated Vehicle Safety Performance at Signalized Intersection". Accident and Accident Prevention, Vol. 40, No. 3, pp. 171-179.

Giles, M.J. (2004). "Driver speed compliance in Western Australia: a multivariate analysis". Transport Policy 11 (2004) 227-235.

Hoogen, E. v., \& Smulders, S. (1994). "Control by Variable Speed Signs: Results of the Dutch Experiment". International Conference on Road Traffic Monitoring and Control (pp. 145-149). London: Institution of Electrical Engineers.

Kwon, E., Brannan, D., Shouman, K., Isackson, C., and Arseneau, B. (2007). “Development and Field Evaluation of Variable Advisory Speed Limit System for Work Zones". Transportation Research Record , 12-18.

Lee, C. and Abdel-Aty, M. (2008). "Testing Effects of Warning Messages and Variable Speed Limits on Driver Behavior Using Driving Simulator". Transportation Research Record: Journal of 
the Transportation Research Board, No. 2069, Transportation Research Board of the National Academies, Washington,D.C., 2008, pp. 55-64.

C. Lee, Hellinga, B., and Saccomanno, F. (2003). "Proactive Freeway Crash Prevention Using RealTime Traffic Control". Canadian Journal of Civil Engineering - Special Issue on Innovations in Transportation Engineering. 30(6). Pages 1034-1041

MODOT (2009). http://www.modot.mo.gov/stlouis/links/VariableSpeedLimits.htm (Accessed August 31, 2009)

Papageorgiou, M., Kosmatopoulos, E., and Papamichail, I. (2008). "Effects of Variable Speed Limits on Motorway Traffic Flow". Transportation Research Record: Journal of the Transportation Research Board, No. 2047, Transportation Research Board of the National Academies, Washington, D.C., 2008, pp. 37-48.

Park, B. and Yadlapati, S.S. (2003). "Development and Testing of Variable Speed Limit Logics at Work Zones using Simulation". Compendium of Papers from the $82^{\text {nd }}$ Annual Meeting of the Transportation Research Board held in Washington D.C.

Povey, L.J., Frith, W.J., and Keall, M.D. (2003). “An investigation of the relationship between speed enforcement, vehicle speeds and injury crashes in New Zealand" Land Transport Safety Authority.

Riffkin, M., McMurtry, T., Heath,S., and Saito, M. (2008). “Variable Speed Limit Signs: Effects on Speed and Speed Variation in Work Zones" Report No. UT-08-01 prepared for the Utah Department of Transportation.

Rodier, C.J., Shaheen, S.A., and Cavanagh, E. (2007). "Automated Speed Enforcement in the U.S.: A Review of the Literature on Benefits and Barriers to Implementation" Compendium of Papers 
from the $87^{\text {th }}$ Annual Meeting of the Transportation Research Board held Jan. 13-17, 2008 in Washington D.C. Paper \# 08-1695.

Transportation Research Board (1998). Special Report 254: Review of Current Practice for Setting and Enforcing Speed Limits. Committee for Guidance on Setting and Enforcing Speed Limits, Transportation Research Board, National Research Council, National Academy Press.

UK Highways Agency. (2006). M25 Controlled Motorway Summary Report. European Commission, Directorate General Energy and Transport.

Ulfarsson, G.F., Shankar, V.N. and Vu, P. (2005). "The effect of variable message and speed limit signs on mean speeds and speed deviations", Int. J. Vehicle Information and Communication Systems, Vol. 1, Nos. 1/2, pp.69-87.

WSDOT (2009). http://www.wsdot.wa.gov/Projects/190/TwoWayTransit/vsl.htm (Accessed July $5,2009)$ 


\section{LIST OF FIGURE CAPTIONS}

Figure 1: Existing Speed Limit Compliance Study Area (Source: Google, 2009)

Figure 2: Free-Flow Speed Profile from QEW

Figure 3: Comparison of criteria used to determine free flow observations

Figure 4: Driver compliance scenarios

Figure 5: Simulation Network

Figure 6: Decision Path for Determining New Posted Speed

Figure 7: VSL performance sensitivity to speed compliance (Average of 10 simulation runs)

Figure 8: Posted speed limit, average vehicle speeds, and detector occupancy for the Very High compliance scenario (Seed: 5; Detector Station 100)

Figure 9: Impact of changing occupancy threshold from 15\% to 20\% (Seed: 5; Detector Station 100)

Figure 10: Impact of changing occupancy threshold from $15 \%$ to $20 \%$ and reducing speed thresholds by $10 \mathrm{~km} / \mathrm{h}$ (Seed: 5; Detector Station 100)

Figure 11: Impact of parameters on VSL performance for the Very High compliance scenario 


\section{TABLES}

Table 1: Speed limit compliance data (Source: Giles, 2004)

\begin{tabular}{|c|c|c|c|c|}
\hline $\begin{array}{c}\text { Posted Speed Limit } \\
(\mathrm{km} / \mathrm{h})\end{array}$ & $\begin{array}{c}\text { Mean Free Flow Speed } \\
(\mathrm{km} / \mathrm{h})\end{array}$ & $\begin{array}{c}\text { Std } \\
(\mathrm{km} / \mathrm{h})\end{array}$ & $\begin{array}{c}\text { COV } \\
\text { Percent Exceeding Posted }\end{array}$ & Speed Limit \\
\hline 60 & 60.9 & 10.7 & 0.18 & 55.7 \\
\hline 70 & 67.7 & 10.3 & 0.15 & 40.7 \\
\hline 80 & 77.3 & 10.7 & 0.14 & 38.4 \\
\hline 90 & 85.4 & 12.8 & 0.15 & 36.9 \\
\hline 100 & 93.8 & 12.6 & 0.13 & 30.2 \\
\hline 110 & 101.7 & 14.3 & 0.14 & 27.4 \\
\hline
\end{tabular}

Table 2: Expected mean free flow speed $(\mathrm{km} / \mathrm{h})$ as a function of speed limit compliance level

\begin{tabular}{|c|c|c|c|c|}
\cline { 2 - 5 } \multicolumn{1}{c|}{} & \multicolumn{4}{c|}{ Compliance Level } \\
\hline Posted Speed Limit & & & & \\
$(\mathrm{km} / \mathrm{h})$ & Low & Moderate & High & Very High \\
\hline 100 & 119 & 119 & 108 & 95 \\
\hline 80 & 104 & 95 & 86 & 76 \\
\hline 60 & 88 & 71 & 65 & 57 \\
\hline
\end{tabular}

